\title{
PRINCIPLE OF OBJECTIVITY AND CLASSICAL PHYSICS
}

\author{
M. DUTTA \\ Satyendranath Bose Institute of Physical Sciences \\ Calcutta University \\ and \\ Centre of Advanced Study in Applied Mathematics \\ Calcutta University \\ U. BASU \\ Department of Applied Mathematics \\ Calcutta University \\ (Received September 1, 1978)
}

ABSTRACT. In 1iterature, it is generally asserted that the "principle of objectivity" is valid for constitutive equations but not valid for general field equations. In this paper, the principle of objectivity is formulated clearly to emphasize the correct significance, and necessity of the principle and to discuss its validity in physical theories. A discussion is made to show how successfully the principle of objectivity is also applicable to non-relativistic classical physics.

KEY WORDS AND PHRASES. Principle of Objectivity, Classical Physics, Vectors, Tensors, Electromagnetic Theory.

AMS (MOS) SUBJECT CLASSIFICATION (1970) CODES. 78A25 
1. INTRODUCTION.

In the theory of mathematical sciences, the main aim is to fit suitable mathematical models to some objects of the objective world with respect to some of its significant aspects. These mathematical models are mentally constructed, and so it is desirable to eliminate subjective factors and factors depending on the state of the observer. Moreover the theories are based on some measurements. Suitably constructed instruments and suitable processes are used. Also attempts are always made to construct mathematical models, i.e., the mathematical formulation of laws indifferent of particular method of measuring process, method of measurements as far as possible. For this some criteria are generally to be introduced to make the theories expressed in similar form or better covariant of the modes of measurement as far as possible. This criterion is generally expressed through the principle of objectivity.

In "Principia", after formulating three general rules, Newton formulated Rule IV which is a criterion to be satisfied by mathematical theories of the objective world.

Einstein (1) also discussed the necessity of some principle of objectivity in the mathematical formulation of physics and tried to give illustrations in his work. The principle of objectivity is clearly formulated 0ldroyd (2), in an article of Truesdell and Toupin (3) in connection with developments of constitutive theory of macroscopic physics treated as continuum physics. But its general significance has been clearly pointed out by Noll (4) and others.

\section{PRINCIPLE OF OBJECTIVITY.}

To understand the principle of objectivity in proper background the observation of Einstein (1) appears to be useful. In this connection mention may be made of the following two statements of Einstein. First, "The laws of physics must be of such a nature that they apply to systems of reference in any kind of motion". Second, "The general laws of nature are to be expressed by 
equations which hold good for all systems of coordinates, that is, are co-variant with respect to any substitution whatever (generally covariant)." An objective law is defined by the rigid transformation,

$$
\underline{x}^{*}=\underline{R}(t) \underline{x}+\underline{b}(t)
$$

where $\underline{x}, \underline{x}^{*}$ are the position-vectors of a point $P$ relative to the origin 0 and $0 *$ respectively in two orthogonal frames; $\underline{b}(t)$ the translation of $*_{\text {-frame relative to }}$ the other frame; $\underline{R}(t)$ is a matrix representing the rotation of the $*$-frame with respect to the other frame i.e. $\underline{R}(t) \underline{R}^{T}(t)=\underline{I}, \underline{R}^{T}(t)$ being the transpose of $\underline{R}(t)$ and $t$ is a parameter representing time. The group of rigid motion is deeply involved in the nonrelativistic classical physics. The group of rigid motion may be written with respect to a frame.

DEFINITION. (i) An objective tensor of rank $k$ is defined by the relation

$$
{ }^{\mathrm{T}}{ }_{\mathrm{i}_{1}} \mathrm{i}_{2} \ldots \ldots \ldots \mathrm{i}_{\mathrm{K}}=\mathrm{R}_{\mathrm{i}_{1} \mathrm{j}_{1}}(\mathrm{t}) \mathrm{R}_{\mathrm{i}_{2} \mathrm{j}_{2}}(\mathrm{t}) \ldots \mathrm{R}_{\mathrm{i}_{k}} \mathrm{j}_{k} \quad(t) \mathrm{T}_{\mathrm{j}_{1}} \ldots \mathrm{j}_{k}
$$

for coordinate transformation given by (2.1) where $\underline{T}$ is a tensor of rank $k$.

(ii) An equation of the form:

$$
\underline{T}_{1}+\underline{T}_{2}+\ldots \ldots+\underline{T}_{n}=0
$$

is said to be objective, when $\underline{T}_{i}$ 's are objective tensors of same rank.

The principle of objectivity states that, every physical equation can be expressed as objective equation.

\section{OBJECTIVE TENSOR ANALYSIS.}

PROPOSITION 1. Time derivative of a position vector is not objective, but there exists another objective vector associated with the time derivative of position vector. 
PROOF. Differentiating (2.1), with respect to time $t$,

$$
\underline{\dot{x}}^{*}=\underline{\dot{R}}(t) \underline{x}(t)+\underline{R}(t) \underline{\dot{x}}(t)+\underline{\dot{b}}(t),
$$

where $\underline{\dot{x}}, \underline{\dot{x}}^{*}$ are the time derivatives of $\underline{x}$ and $\underline{x}^{*}$ in the respective frames. It is easily found that $\underline{\dot{x}}$ is not an objective vector.

Similarly the second order time derivative of $\underline{x}$ in the respective frame is not an objective vector.

Now it will be shown that corresponding to the time derivative of postion vector there exists another vector which is objective.

Let $\underline{x}_{I}$ be the position vector with respect to a frame $I$ at absolute rest. Considering a coordinate transformation, one has

$$
\underline{x}=\underline{R}_{I}(t) \underline{x}_{I}(t)+\underline{b}_{I}(t),
$$

where $\underline{R}_{I}(t)$ and $\underline{b}_{I}(t)$ are the rotation and translation of the new frame with respect to the inertial frame.

Differentiating (3.2) with respect to time $t$, it follows that

$$
\underline{\dot{x}}=\underline{\dot{R}}_{I}(t) \underline{x}_{I}+\underline{R}_{I}(t) \underline{\dot{x}}_{I}+\underline{\dot{b}}_{I}(t),
$$

or

$$
\underline{\dot{x}}_{I}=\underline{R}_{I}^{T}(t)\left\{\underline{\dot{x}}-\underline{\dot{R}}_{I}(t) \underline{R}_{I}^{T}(t) \quad(\underline{x}-\underline{b}(t))-\underline{\dot{b}}_{I}(t)\right\} \text {, }
$$

Let

$$
\underline{v}=\underline{\dot{x}}-\underline{\dot{R}}_{I}(t) \underline{R}_{I}^{T}(t)(\underline{x}-\underline{b}(t))-\underline{\dot{b}}_{I}(t),
$$

and

$$
\underline{\mathrm{v}}_{\mathrm{I}}=\dot{\mathrm{x}}_{\mathrm{I}}
$$

Therefore it follows from (3.4) that,

$$
\underline{\mathrm{v}}=\underline{\mathrm{R}}_{\mathrm{I}}(\mathrm{t}) \underline{\mathrm{v}}_{\mathrm{I}}
$$


Let the frame at absolute rest coinciding instantaneously at time $t$ with the frame in which $\underline{x}$ is the position vector be denoted by $I(t)$. It is easy to see that

$$
\underline{v}_{I(t)}(t)=\left[\underline{R}_{I}(t)\right] \quad \underline{v}_{I}
$$

where $\underline{R}_{I}(t)$ is the instantaneous value at time $t$.

Thus, $\mathbf{v}$ may be interpreted as the velocity relative to the frame at absolute rest instantaneously coinciding with the moving frame in which the position vector is represented by $\underline{x}(t)$.

To show that this velocity $\underline{v}$ is objective, a *-frame is introduced by

$$
\underline{x}^{*}(t)=\underline{R}_{I}^{*}(t) \underline{x}_{I}+\underline{b}_{I}^{*}(t)
$$

Then

$$
\underline{x}^{*}=\underline{R}(t) \underline{x}(t)+\underline{b}(t)
$$

where

$$
\underline{R}(t)=\underline{R}_{I}^{*}(t) \cdot \underline{R}_{I}^{T}(t), \underline{b}(t)=\underline{b}_{I}^{*}(t)-\underline{R}_{I}^{*}(t) \underline{R}_{I}^{T}(t) \underline{b}_{I}(t) \cdot
$$

Now, $\quad \underline{v}_{I}(t)=\underline{R}_{I}^{T}(t) \underline{v}(t)=\underline{R}^{*} I(t) \underline{v}^{*}(t)$

i.e.

$$
\underline{v}^{*}(t)=\underline{R}_{I}^{*}(t) \cdot \underline{R}_{I}^{T} \underline{v}(t)=\underline{R}(t) v(t)
$$

Thus $\underline{\mathrm{v}}$ is an objective vector.

After evaluating $\underline{\dot{R}}(t) \cdot \underline{R}^{T}(t) \underline{x}$, it has been easily obtained that

$$
\underline{\dot{\mathrm{R}}}(\mathrm{t}) \underline{\mathrm{R}}^{\mathrm{T}}(\mathrm{t}) \underline{\mathrm{x}}=-\underline{\Omega} \times \underline{\mathrm{x}}
$$

where $\underline{\Omega}$ is the rotation of one coordinate system about the fixed system and $\underline{R}(t)$ is the coordinate-transformation matrix.

Hence from (3.5), it follows that

$$
\underline{v}=\left(\underline{\dot{x}}-\underline{b}_{I}(t)\right)+\underline{\Omega} \times\left(\underline{x}-\underline{b}_{I}(t)\right)
$$

Now $\underline{v}$ is called the total derivative of position vector. Hence the total derivative 
is objective. It is clear from any book of classical mechanics Goldstein (5), that $\underline{v}$ is the velocity of the point. Then it follows that velocity is objective. So following propositions follow:

PROPOSITION 1. Velocity is an objective vector.

PROPOSITION 2. If $\underline{P}(t)$ is an objective vector, then its time derivative is not so but its total derivative is so.

PROOF. If $\underline{\Omega}$ is the rotation of a new frame with respect to the fixed frame, then total derivative of any vector $\underline{P}(t)$ is defined by

$$
\frac{D \underline{P}(t)}{D t}=\underline{\dot{P}}(t)+\underline{\Omega} \times \underline{P}(t)
$$

Since $\underline{P}(t)$ is an objective vector, under transformation $(2.1) \underline{P}(t)$ changes to $\underline{P} *(t)$, then

$$
\underline{P}^{*}(t)=\underline{R}(t) \underline{P}(t)
$$

Differentiation with respect to $t$ gives

$$
\underline{\dot{P}}^{*}(t)=\underline{R}(t) \underline{P}(t)+\underline{\dot{R}}(t) \underline{P}(t)
$$

where $\underline{\dot{p}}(t)$ and $\underline{\dot{p}}^{*}(t)$ are time derivatives of $\underline{\underline{p}}$ and $\underline{P}^{*}$. It is clear that $\underline{\dot{P}}(t)$ is not an objective vector.

It will be shown that with the time derivative of an objective vector, another vector can be associated which is an objective vector. Consider the following two rigid transformations

$$
\begin{aligned}
& \underline{x}_{1}(t)=\underline{Q}_{1}(t) \underline{x}_{0}(t)+\underline{b}_{1}(t), \\
& \underline{x}_{2}(t)=\underline{Q}_{2}(t) \underline{x}_{0}(t)+\underline{b}_{2}(t),
\end{aligned}
$$

where, $\underline{Q}_{1}, \underline{Q}_{2}$ are the coordinate transformation matrices and let $\Omega_{1}, \underline{\Omega}_{2}$ be the 
rotations of the new coordinate systems about fixed $x_{0}$-system of coordinates. If $\underline{P}_{0}(t)$ changes to $\underline{P}_{1}(t), \underline{P}_{2}(t)$ in the rotating systems then

$$
\begin{aligned}
& \underline{P}_{1}(t)=Q_{1}(t) \quad \underline{P}_{0}(t), \\
& \underline{P}_{2}(t)=\underline{Q}_{2}(t) \underline{P}_{0}(t),
\end{aligned}
$$

It follows from (3.16) and (3.17) that

$$
\underline{\mathrm{P}}_{2}(t)=\underline{Q}_{2}(t) \underline{Q}_{1}^{-1}(t) \underline{P}_{1}(t)=\underline{R}^{(t)} \underline{P}_{1}(t) \text { (say) }
$$

Differentiation of (3.16) and (3.17) with respect to $t$, give

$$
\begin{aligned}
& \dot{\underline{p}}_{1}(t)=\dot{Q}_{1}(t) \underline{p}_{0}(t)+\underline{Q}_{1}(t) \stackrel{\dot{p}}{0}_{0}(t), \\
& \dot{\underline{p}}_{2}(t)=\dot{Q}_{2}(t) \underline{p}_{0}(t)+\underline{Q}_{2}(t) \dot{\dot{p}}_{0}(t),
\end{aligned}
$$

Again

$$
\begin{aligned}
& \underline{Q}_{1}(t) \dot{p}_{0}(t)=\dot{p}_{1}(t)-\dot{Q}_{1}(t) \underline{Q}_{1}^{-1}(t) \underline{p}_{1}(t)=\dot{p}_{1}(t)+\underline{\Omega}_{1} \times \underline{p}_{1}, \\
& \underline{Q}_{2}(t) \underline{\dot{p}}_{0}(t)=\dot{p}_{2}(t)-\dot{q}_{2}(t) \underline{Q}_{2}^{-1}(t) \underline{p}_{2}(t)=\dot{p}_{2}(t)+\underline{\Omega}_{2} \times \underline{P}_{2},
\end{aligned}
$$

Hence it is clear from (3.21) and (3.22) that

$$
\begin{aligned}
& \underline{Q}_{1}(t) \dot{p}_{0}(t)=\dot{P}_{1}(t)+\underline{\Omega}_{1} \times \underline{P}_{1}=\underline{v}_{1}^{P} \quad \text { (say), } \\
& \underline{Q}_{2}(t) \underline{\mathrm{p}}_{\mathrm{o}}(t)=\underline{\mathrm{P}}_{2}(t)+\underline{\Omega}_{2} \times \underline{\mathrm{P}}_{2}=\underline{\mathrm{v}}_{2}^{\mathrm{P}} \quad \text { (say), } \\
& \text { and } \dot{\dot{p}}_{0}=\underline{\mathrm{v}}_{\mathrm{v}}^{\mathrm{P}}, \quad \text { (say), }
\end{aligned}
$$

i.e. $\quad \underline{v}_{2}^{P}(t)=\underline{Q}_{2}(t) Q_{1}^{-1}(t) \quad \underline{v}_{1}^{P}(t)=Q_{2}(t) \underline{v}_{0}^{P}(t)$

This result proves that $\underline{v}_{1}^{\mathrm{P}}, \underline{\mathrm{v}}_{2}^{\mathrm{P}}$ are objective i.e. the total derivative of an objective vector is objective. 
Now from (3.18), (3.21) and (3.22) one gets

$\underline{\dot{P}}_{2}(t)+\left\{\left(\underline{\Omega}_{2} \times\right)-\underline{Q}_{2}(t) \underline{Q}_{1}^{-1}(t)\left(\underline{\Omega}_{1} \times\right)\left(\underline{Q}_{2}(t) \underline{Q}_{1}^{-1}(t)\right)^{-1}\right\} \underline{P}_{2}(t)=Q_{2}(t) Q_{1}^{-1}(t) \dot{P}_{1}$.

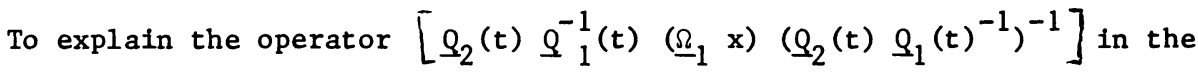
expression (3.27), it is necessary to introduce symmetry operators. Let $\underline{A}$ be an operator in space such that $\underline{Z}=\underline{A} \underline{Y}$. Now there is a coordinate transformation defined by

$$
\underline{Y}^{\prime}=\underline{R} \underline{Y} \quad, \quad \underline{Z}^{\prime}=\underline{R} \underline{Z}
$$

If $\underline{A}^{-}$is the operator such that $\underline{Z}^{\prime}=\underline{A}^{\prime} \underline{Y}^{\prime}$, then $\underline{A}^{\prime}=\underline{R} \underline{A} \underline{R}^{-1}$

and $\underline{A}^{\prime}$ is called the symmetry operator.

If the operation $\left(\underline{\Omega}_{1} x\right)$ in $(3.27)$ is denoted by operator $\underline{A}$ and coordinate transformation matrix is given gy $\left(Q_{2}(t) \quad Q_{1}^{-1}(t)\right)$ then one gets

$$
\left.\underline{A}^{\prime}=\left(\underline{Q}_{2}(t) \underline{Q}_{1}^{-1}(t)\right) \quad \underline{A}_{2}(t) \underline{Q}_{1}^{-1}(t)\right)^{-1}
$$

Then $\underline{A}^{\prime}$ can be replaced by the operator of the type $\left(\Omega_{1}^{\prime} x\right)$. Then from (3.27), (3.29) one gets

$$
\underline{R}_{\underline{P}_{1}}=\dot{\mathrm{P}}_{2}(t)+\left(\underline{\Omega}_{2}-\underline{\Omega}_{1}^{\prime}\right) \times \underline{\mathrm{P}}_{2}
$$

where

$$
\underline{R}=\underline{Q}_{2}(t) \underline{Q}_{1}^{-1}(t) \text {. }
$$

Let

$$
\underline{\Omega}^{-} \underline{\Omega}_{2}-\underline{\Omega}_{1}^{\prime}
$$

It is easy to see that the rotation $\underline{\Omega}$ is the angular velocity of the second frame relative to the first frame (1-frame). In this case it is noted that the relative angular velocity of the frame is not a simple difference $\Omega_{2}-\Omega_{1}$, $\Omega_{2}, \underline{\Omega}_{1}$, being the relation about the fixed frame, but really it is $\underline{\Omega}_{2}-\underline{\Omega}_{1}$ 
where $\Omega_{1}^{\prime}$ is the effect of $\Omega_{1}$ as it will be seen from second rotating frame. This point appears to be new in significance.

COROLLARY. Acceleration is an objective vector.

PROOF. Velocity is an objective vector. If acceleration is defined by the total derivative of the velocity vector then

$$
\underline{\mathbf{f}}=\underline{\dot{v}}+\underline{\Omega} \times \underline{\mathrm{v}} .
$$

In view of proposition - 2, acceleration is obviously an objective vector.

Since elements of the transformation matrix are functions of time, in case of differentiation with respect to space coordinates, elements of transformation matrix behave like constants. Thus follow the propositions stated below.

PROPOSITION 3. Gradient operator is an objective operator.

1.e. $\underline{\nabla}^{*}=\underline{R} \underline{\nabla}$ under the coordinate transformation (2.1).

PROOF. From (2.1) 1t follows that

$$
\frac{\partial}{\partial x_{1}^{*}}=R_{1 j} \frac{\partial}{\partial x_{j}} \text { i.e. } \underline{\nabla} *=\underline{R} \underline{\nabla}
$$

Hence the proposition,

PROPOSITION 4. Divergence of an objective vector is a scalar.

PROOF. Let $\underline{A}$ be an objective vector, then under transformation (2.1)

$$
\begin{aligned}
& \underline{A}^{*}=\underline{R} \underline{A}, \\
& \frac{\partial}{\partial x_{j}^{\star}}\left(A_{j}^{*}\right)=\frac{\partial A_{k}}{\partial x_{k}} \quad \text { (since } R \text { is an orthogonal matrix) }
\end{aligned}
$$

Th1s proves the proposition.

PROPOSITION 5. Any derivative of an objective tensor of rank (k) with respect to space coordinate is an objective tensor of rank (k-1). 
PROOF. Under coordinate transformation (2.1)

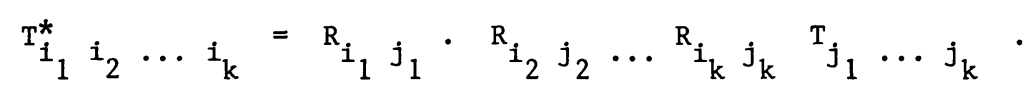

Then

$$
\begin{aligned}
\frac{\partial T_{i_{1} i_{2}}^{*}}{\partial x_{i_{k}}^{*}} \cdots i_{k} & =R_{i_{k}} j_{n} R_{i_{1}} j_{1} \ldots R_{i_{k}} j_{k} \frac{\partial T j_{1} \cdots j_{k}}{\partial x_{j}} \\
& =R_{i_{1}} j_{1} \ldots R_{i_{k-1}} j_{k-1} \frac{\partial T_{j} \cdots j_{k}}{\partial x_{j_{k}}}
\end{aligned}
$$

Hence it is objective tensor of rank $(k-1)$.

PROPOSITION 6. The product of two objective vectors is an objective vector.

PROOF. Let $\underline{A}$ and $\underline{B}$ be two objective vectors. Under transformation (2.1), $\underline{A}, \underline{B}$ change to $\underline{A}^{*}$ and $\underline{B} *$ respectively.

$$
\underline{A}^{*} \times \underline{B}^{*}=e_{i j k}^{*} A^{*}{ }_{j}^{B^{*}}{ }_{k}=R_{i i_{1}} e_{i_{1} j_{1} k_{1}} A_{j_{1}} \quad B_{k_{1}}=\underline{R}(\underline{A} \times \underline{B})
$$

Hence the proposition follows.

PROPOSITION 7. Curl of an objective vector is an objective vector.

$$
\begin{aligned}
& \text { PROOF. Cur1 } \underline{A}^{*}=e_{i j k}^{*} \frac{\partial}{\partial x_{j}^{*}} A^{*}=R_{i i_{1}} e_{i_{1} j_{1} k_{1}} \frac{\partial A_{1}}{\partial x_{j}} \\
& \therefore \quad \operatorname{Cur} 1 * \underline{A}^{*}=\underline{R} \operatorname{Cur} 1 \underline{A} \text {. }
\end{aligned}
$$

This proves the proposition.

\section{OBJECTIVITY IN CLASSICAL MECHANICS OF SYSTEM OF PARTICLES, RIGID BODIES AND DEFORMABLE BODIES.}

It is observed that the Newtonian classical non-relativistic mechanics may be elegantly formulated by using the principle of objectivity. Unfortunately in the literature the objectivity of the equations of balance of mass, momentum, etc., of mechanics has not been noticed properly.

(i) Objectivity in Mechanics of System of Particles: 
The momentum balance equation (Newton's equation of motion) is :

The rate of change of momentum of particle is equal to the impressed force on it.

For a system of particles the equation of motion of $1^{\text {th }}$ particle is

$$
\mathrm{m}_{i} \underline{\mathrm{f}}_{\mathrm{i}}=\underline{\mathrm{F}}_{\mathrm{i}}=\underline{\mathrm{F}}
$$

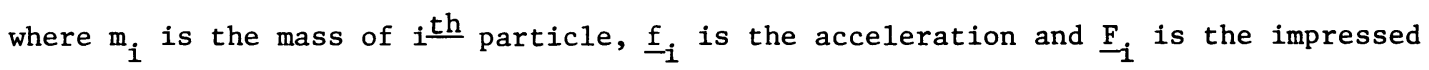
force on it.

The acceleration $\underline{f}_{i}$ involved in (4.1) is an objective vector when it is written in terms of total derivative of velocity vector. Since the mass $m_{i}$ of any particle is independent of any state of motion, $m_{i}$ is a scalar. Then $m_{i} \underline{f}_{i}$ is an objective vector. Now the form of equation of the balance of linear momentum (i.e. Newton's equation of motion) is objective if impressed forces are introduced as objective entities.

Impressed forces are supposed to be forces of interaction with rest of the Universe. These interactions are proportional to the relative distances. These relative distances are obviously objective as they are independant of the translatory motion of reference frames. Some other authors define force as mass times acceleration. In this case force is objective. Even according to other school without any motion, the existence of force may be experienced by senses. When a heavy body rests on hand, then it has no motion and acceleration but its weight can be felt through the muscles and nerves of the hand and so force should be considered something of objective existence and as such may be taken as an objective vector.

Thus the form of equation of the balance of momentum for a system of particles is objective.

In classical theory of relativity of Galileo and Newton, if two frames of reference move with uniform motion then in Newtonian mechanics physical laws are independent of the translatory motion of frame. This is a simple version of the 
principle of objectivity. According to the above principle uniform translatory motion and motions of other types (i.e. non-uniform translatory motion or rotatory motion) have different roles. In the general principle of objectivity as formulated above non-uniform translatory motion do not affect the physical laws. So the principle of objectivity may be referred as some principle wider than the principle of relativity in classical mechanics.

(ii) Objectivity for Motion of Rigid Body :

It is well known that motion of a rigid body is equivalent to the motion of the centre of mass with the entire mass $M$ concentrated at the centre of mass and all external forces transferred to it and the motion of the body about the centre of mass. Let $\mathrm{m}_{\mathrm{P}}$ be the mass at the point $\mathrm{P}$.

Equations of motion can be written as

$$
\begin{aligned}
& \underline{M f_{G}}=\underline{F}_{G}, \\
& \sum \underline{m}_{P} \underline{r}_{G P} \times \underline{f}_{\underline{P}}=\sum \underline{r}_{G P} \times \underline{F}_{\underline{P}}
\end{aligned}
$$

Where $G$ is the centre of mass, $\underline{r}_{G P}$ is the distance of the centre of mass from any point $P$ of rigid body. $\underline{f}_{G}$ and $\underline{f}_{P}$ are accelerations of the point $G$ and $P$ respectively and $\underline{F}_{P}, \underline{F}_{G}$ are the forces acting at the point $P$ and $G$ respectively. So far as the motion of the centre of mass is concerned it is governed by the objective equations of classical mechanics of the particles. For motion about the centre of mass, the objectivity of vectors about the centre of mass should be observed in equation (4.3). $\underline{r}_{G P}$ is objective under the rigid transformation (2.1). Since vector product of any two objective vectors is objective, the form of the equation of motion about the centre of mass is objective.

(iii) Objectivity in Continuum Mechanics :

As objectivity of equations in constitutive theory are discussed in details in the literature it is not necessary to repeat them here. 
Only balance equations will be discussed here.

The balance equation of mass is

$$
\frac{\partial \rho}{\partial t}+\frac{\partial}{\partial x_{k}}\left(\rho v_{k}\right)=0
$$

Here $\rho$ is generally taken as a scalar, so time derivative of $\rho$ is objective. Also divergence of objective vector $\rho \underline{\underline{v}}$ is an objective scalar then under rigid transformation defined by (2.1) balance equation of mass is objective.

REMARK. The equation of balance of mass is sometimes written in a form

$$
\frac{\partial \rho}{\partial t}+\frac{\partial}{\partial x_{k}}\left(\rho \dot{x}_{k}\right)=0
$$

and the form is not objective.

Balance of momentum :

Cauchy's law of balance of momentum should be written as

$$
t_{i k, 1}+(\underline{f}-\underline{F})=0 \text {. }
$$

Here stress tensor $t_{i k}$ is an objective tensor.

Derivative of an objective tensor with respect to space coordinates is an objective vector. Acceleration and impressed force are objective vectors. So the form of balance equation is objeetive.

\section{OBJECTIVITY IN BOLTZMANN EQUATION IN KINETIC THEORY.}

The Boltzmann equation is

$$
\frac{\partial f_{i}}{\partial t}=-\underline{v}_{i} \cdot \frac{\partial f_{i}}{\partial \underline{r}}-\underline{F}_{i} \cdot \frac{\partial f_{i}}{\partial \underline{v}_{i}}+\sum_{j} c\left(f_{i}, f_{j}\right), i=1, \ldots N
$$

where $f_{i}$ is the distribution function which describes the behavior of the system, $\underline{F}_{1}$ is an external force (per unit mass) on the component $1, \underline{v}_{1}$ is the velocity of 
component $i$, and $C\left(f_{i}, f_{j}\right)$ are collision integrals.

Classical (Newtonian) mechanics provides the basis of kinetic theory. As the basic equation of Newtonian mechanics is of second order, so along with the initial position, initial velocity are arbitrary. Then at any instant the state of motion of a particle of classical mechanics is completely specified by ( $x, y, z)$ and $\left(v_{x}, v_{y}, v_{z}\right)$ where $v_{x}, v_{y}, v_{z}$ are generally taken to be independent of $x, y, z$ but are parallel to their directions. So $\left(v_{x}, v_{y}, v_{z}\right)$ is to be taken as an objective vector. So $\nabla_{\underline{v}}$ is also an objective operator as the coordinate axes are orthogonal to each other. It is ahead shown that acceleration is also an objective vector.

Here $f_{i}$ is the distribution function and so the number density $f_{i} d x d y d z d v_{x} d v_{y} d v_{z}$ is a scalar. Again $d x d y d z d v_{x} d v_{y} d v_{z}$ is a scalar, so $f_{i}$ is a scalar. Therefore $f_{i}$ is objective and $\frac{\partial f_{i}}{\partial t}$ in (5.1) is objective. Since mass is a scalar and hence mass times acceleration is an objective vector. Again $\underline{F}_{i}=\underline{m a}_{i}$, where $\underline{a}_{i}$ is the acceleration of component $i$. Therefore $\underline{F}_{i}$ is an objective vector. So $\underline{v}_{i} \cdot \nabla_{\underline{r}} f_{i}$ and $\underline{F}_{i} \cdot \nabla_{\underline{v}} f_{i}$ are scalars. Then form of the basic equations of kinetic theory is objective.

Like other cases, this equation is not independent or dependent on the frame of reference of the observer but really is covariant as this is already stated.

\section{OBJECTIVITY IN ELECTROMAGNETIC THEORY.}

With standard notations, the Maxwell's equations are

$$
\begin{aligned}
& \underline{\nabla} \cdot \underline{E}=4 \pi \rho, \\
& \underline{\nabla} \cdot \underline{B}=0, \\
& \underline{\nabla} \times \underline{E}=-\left(\frac{1}{c}\right) \frac{\partial B}{\partial t}, \\
& \underline{\nabla} \times \underline{B}=4 \pi j+\frac{1}{c} \frac{\partial E}{\partial t},
\end{aligned}
$$

where $\rho$ is the charge density, $j$ the current density, $c$ the velocity of light, $\underline{E}$ is the electric field and $\underline{B}$ is the magentic field. 
Now $\underline{B}$ and $\underline{E}$ are objective vectors and $c$ is a scalar. So the form of equations (6.1) and (6.2) is objective.

In the right hand side of equations (6.3) and (6.4), in place of the partial derivatives of $\underline{B}$ and $\underline{E}$ with respect to time we have to take the total derivatives of $\underline{B}$ and $\underline{E}$ with respect to time. According to the Faraday's laws of induction for a moving observer even the static electric or magnetic field will appear as general electromagnetic field. Also the form of the equations (6.3) and (6.4) are objective.

\section{CONCLUDING REMARKS.}

The above entire discussions have been confined to classical non-relativistic physics. The principle of objectivity is formulated with respect to measurements by the rigid rod. But, when relativistic physics is introduced, measurement by the light signal is to be admitted and hence so formulation of the principle of objectivity is to be modified suitably. This will be studied in future.

In classical relativistic and also in non-relativistic physics, it is the implicit assumption that measurement process does not disturb the objects measured. In quantum physics measurement process disturbs objects measured. So the entire principle is to be reformulated and modified suitably from basic consideration.

\section{REFERENCES}

1. Einstein, A., "The Generalized Principle of Relativity" English translation of the Principle of Relativity, Dover Series, New York (1916).

2. Oldroyd, J.G., "On the Formulation of Rheological Equation of State" Proc. Roy. Soc. London 200 (1950) 523-541.

3. Truesde11, C. and R.A., Toupin, "The Classical Field Theories" Handbuch Der Phisik Vol. 111/1 Springer-Verlag, Berlin (1960).

4. Noll, W., "The Axiomatic Method" Edited by Henkin, L. Suppes, P. and Tarski, A. North Holland Publishing Company, Amsterdam (1951) 226.

5. Goldstein, H., "Classical Mechanics" Addison Wesley Press, Inc. Cambridge, Mass. 42 (1950). 


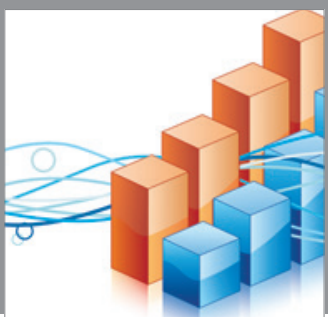

Advances in

Operations Research

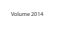

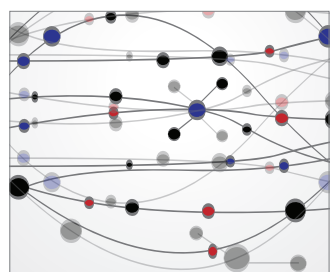

\section{The Scientific} World Journal
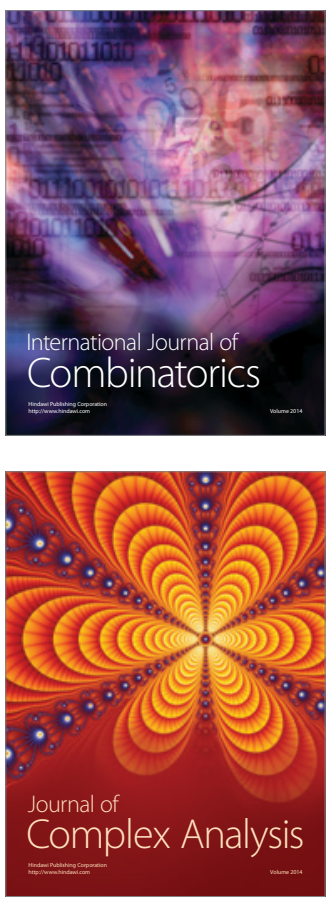

International Journal of

Mathematics and

Mathematical

Sciences
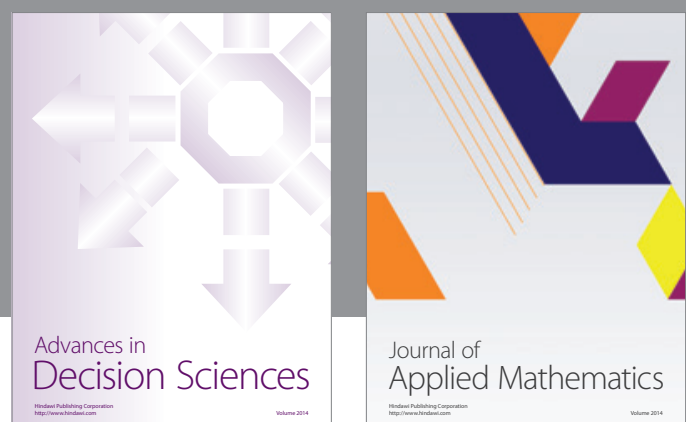

Journal of

Applied Mathematics
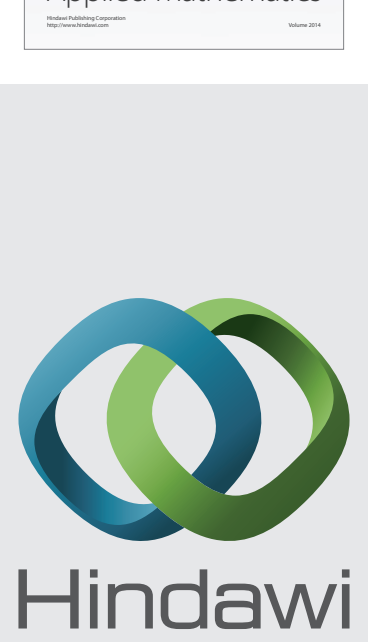

Submit your manuscripts at http://www.hindawi.com
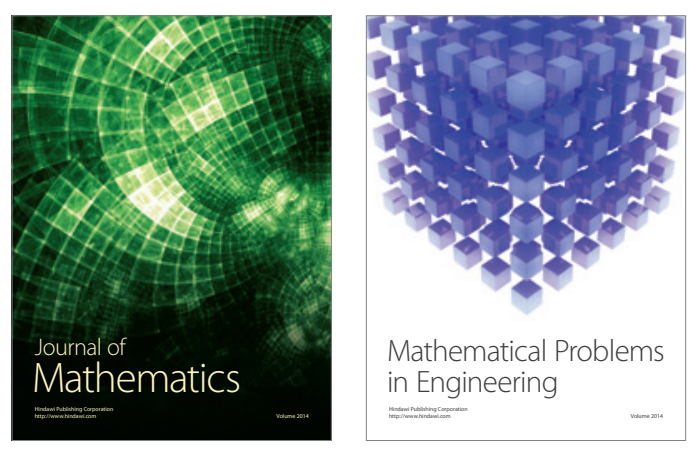

Mathematical Problems in Engineering
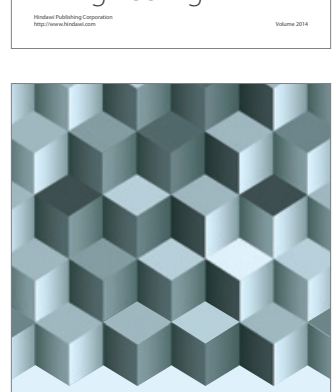

Journal of

Function Spaces
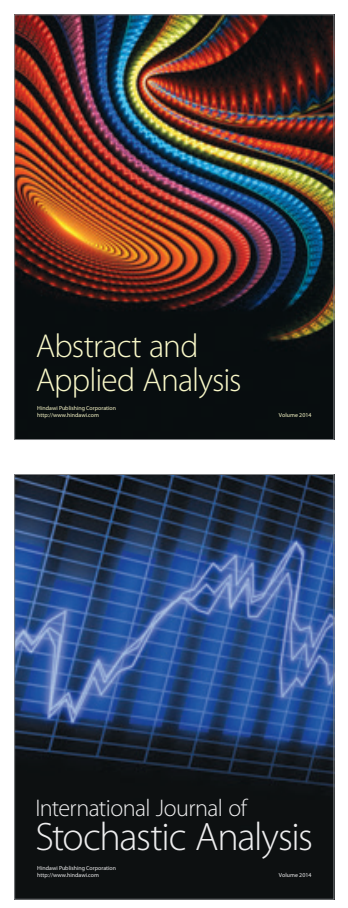

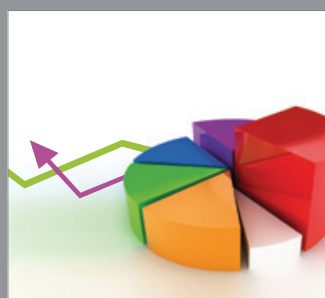

ournal of

Probability and Statistics

Promensencen
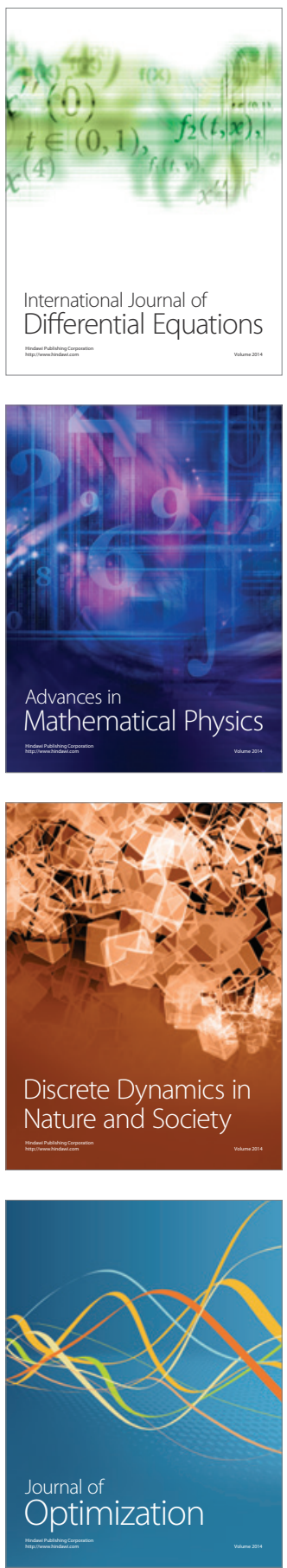